\title{
AUTOMATED ATTENDANCE SYSTEM WITH RFID THROUGH SMART CARD
}

\author{
Miss.Namrata D. Kadapure \\ Student \\ Dept. Of Computer Science \& Engineering \\ ATS's ,SBGI, Miraj \\ Mrs.S G Bavachkar \\ Assistant. Professor \\ Dept. Of Computer Science \& Engineering \\ ATS's ,SBGI, Miraj
}

\begin{abstract}
Understudy participation is one of the significant issues for universities, on the grounds that numerous schools assess understudies' participation and keeping in mind that giving the last grade, educators think about their all-out number of appearances on classes during the entire semester. A few universities like to utilize paper sheet for controlling participation, while a few schools like to utilize paper sheet for checking understudies' participation and after this, round out these data into a framework physically Generally understudies' exhibition in examinations is relying upon the participation. There is a need to foster framework that diminishes trouble in examining the participation and upgrade smooth working of schools, universities and schools and to help the guardians. In our undertaking portrays a short prologue to utilization of participation framework and surveys some use of participation framework and strategies of information recovery like keen card, biometrics and RFID itself. This framework would be material in gathering understudy participation in homeroom utilizing aloof RFID innovation. Our task is Monitoring of Student utilizing RFID. RFID represents Radio Frequency Identification and Detection. In this we are utilizing RFID peruser and contactless shrewd card.
\end{abstract}

Keywords-feedback, management, system, project, Educational

\section{INTRODUCTION}

In classrooms, time is squandered in roll calls as it is done physically. In this proposed framework, approved understudy is given a RFID tag. Once the student places the card in front of the RFID card reader, it reads the data and verifies it with the data stored in the microcontroller from 8051 family. The traditional method of monitoring has some drawbacks. This method obviously not efficient as it wastes the user's energy and quite slow in term of completion. For example, a class that

\author{
Miss.Tasnim R. Jamadar \\ Student \\ Dept. Of Computer Science \& Engineering \\ ATS's ,SBGI, Miraj \\ Miss.Sneha A. Vharagar \\ Student \\ Dept. Of Computer Science \& Engineering \\ ATS's ,SBGI, Miraj
}

uses attendance sheet method requires the students to pass the sheet to each other to sign up the monitor.

\section{EXISTING SYSTEM}

In many colleges, instructors gauge participation by getting down on the names and family names of understudies, and the stamping them, while, in others, educators pass around a piece of paper, requesting that understudies sign in participation sheet only close to their last names. The two practices have the disadvantages. In the principal case, if various gatherings go to the exercise, checking these understudies by name and last name may remove around a little way from every exercise; in the subsequent case, companions of missing understudies may record their names and family names. These practices plan college instructors and their organizations at impressive inconveniences with regards to gauging participation. Older style understudy participation framework was a dreary errand to perform and keep up and furthermore tedious standardized identifications are less secure on the grounds that can be effortlessly recreated. standardized tag innovation is strategy to ID which is utilized to recover fit as a fiddle of image commonly in bar, vertical, space, square which have distinctive width and tallness every one peruser can distinguish information that are addressed by utilizing light pillar and sweep diversely yet during filtering more information are lost.

\section{PROPOSED SYSTEM}

We created computerized understudy participation framework RFID box SMART CARD framework we improve the understudy observing framework. Use RFID and savvy card. With the assistance of that improve security. There will RFID transponders introduced in each homeroom. What's more, when an understudy enters any of them the transponder will distinguish and store the understudy's last known situation in the information base. The total cycle will be robotized and nobody should screen the framework. As each tag has its own 
remarkable ID, it is not difficult to separate each label holder. This is interestingly understudy distinguish. We oversee participation data set. Assuming understudy are absent, send the message to watchman. This framework utilizes individual RFID labels for every client recognizable proof.

Advantages of Proposed System:

- Reduces a lot of time and effort

- Reduces paper work

- Friendly user interface

- Enhances security

\section{PROBLEM STATEMENT}

In every class it is not possible to get the attendance of every student. It is more time consuming so because of this online attendance system is Day-to-Day requirements.

\section{OBJ ECTIVE}

- Online attendance system is more effective than traditional attendance system.

- The Arduino base micro controller unit is $\mathrm{i} / \mathrm{f}$ with $\mathrm{rf}$ reader and wi-fi module to calculate the attendance and upload to online web portal.

- The main objective is the IoT base online portal which can check daily individual attendance.

- The system is more effective in handling and rf reader card can easily swiped.

\section{SYSTEM REQUIREMENTS}

A) Hardware Components

B) Software Requirement

\section{A) Hardware Components:}

Hardware components include Arduino, LCD, Wi-Fi module, RFID reader EM-18, Web server.

\section{Arduino:}

The Arduino UNO is the best board to get started with electronics and coding. If this is your first experience tinkering with the platform. The UNO is the most used and documented board of the whole Arduino family.

\section{LCD:}

A LCD is a flat panel display. Liquid crystals do not emit light directly. LCDs are available to display arbitrary images.

\section{Wi-Fi Module:}

The ESP8266 Wi-Fi module is a self-contained SOC. With TCP/ IP protocol stack. ESP8266 is capable of either hosting an application, offloading all Wi-Fi networking.

\section{EM18 RFID reader:}

RFID is the wireless non-contact use of radio frequency waves to transfer data. Tagging items with RFID tags allows users to automatically and uniquely identify and track inventory and assets. RFID takes autoID technology to the next level by allowing tags to be read without line of sight and depending on the type of RFID, having a read range between a few centimeters.

\section{Web server:}

A web server is software and hardware that used HTTP and other protocols TP respond to client requests made over the world wide web. The main job of web server is to display website content through storing, processing and delivering web pages to users.

\section{B) Software Requirements:}

Software requirements include Arduino C compiler, HTML, PHP.

\section{HTML:}

HTML (Hypertext Markup Language) is the code that is used to structure a web page and its content. For example, content could be structured within a set of paragraphs, a list of bulleted points, or using images and data tables. As the title suggests, this article will give you a basic understanding of HTML and its functions.

\section{PHP:}

PHP is a recursive acronym for "PHP: Hypertext Preprocessor".PHP is a server-side scripting language that is embedded in HTML. It is used to manage dynamic content, databases, session tracking, even build entire ecommerce sites. It is integrated with a number of popular databases, including MySQL, PostgreSQL, Oracle, Sybase, Informix, and Microsoft SQL Server. 


\section{SYSTEM ARCHITECTURE}

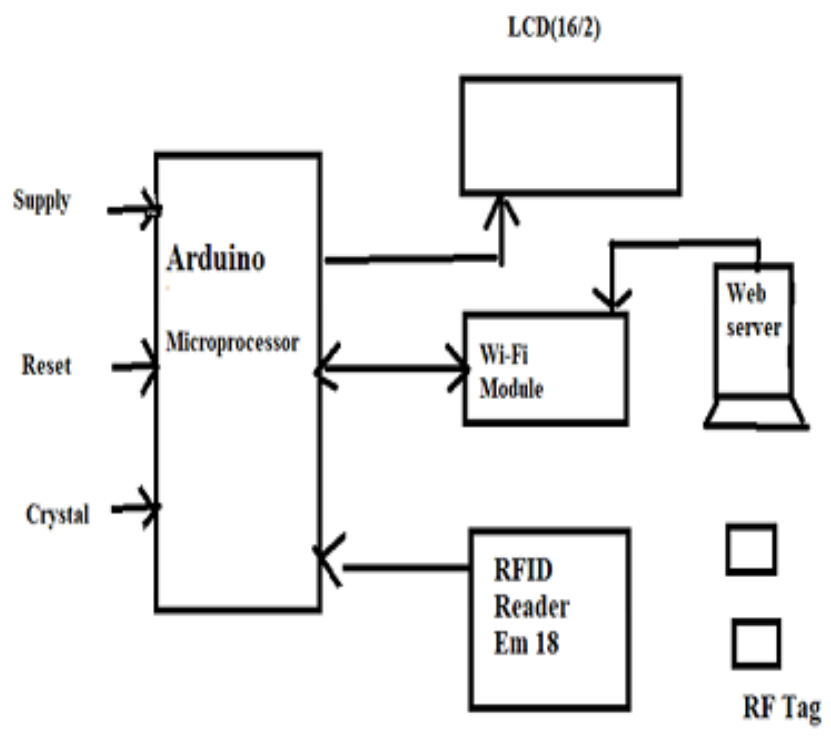

Figure 7.1 System Architecture

\section{Methodology Used:}

1. Arduino based microcontroller unit is required to handle on input output devices.

2. Arduino is interface with reference reader which is having 12-digit binary code for a unique user.

3. After swiping the card the digit code is read by EM18 reader.

4. It is serially transmitted to Arduino and display on LCD i.e. Roll-no and Name.

5. The Wi-Fi module is interfaced with Arduino to upload this attendance to web server.

6. The web server is designing HTML and PHP.

\section{WORKFLOW OF SYSTEM}

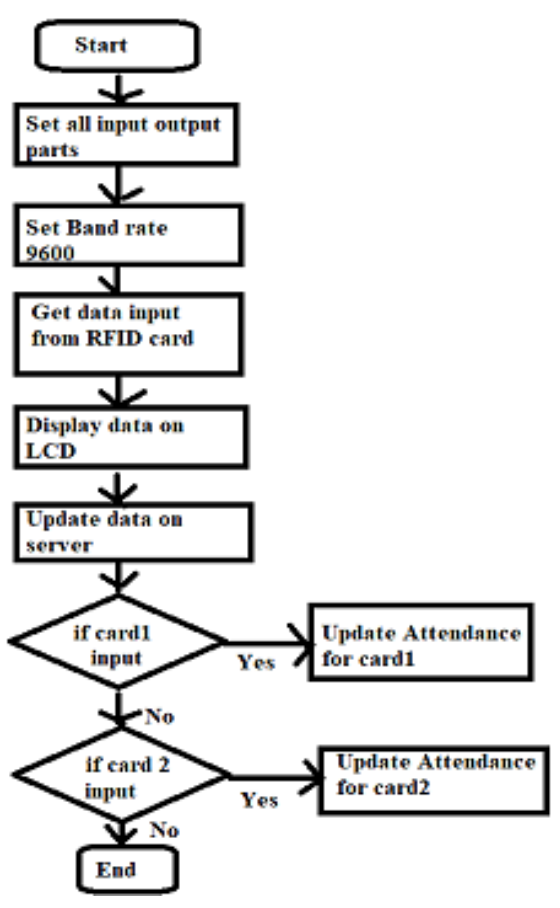

Fig: 8.1 Workflow of system

IX. RESULT ANALYSIS:

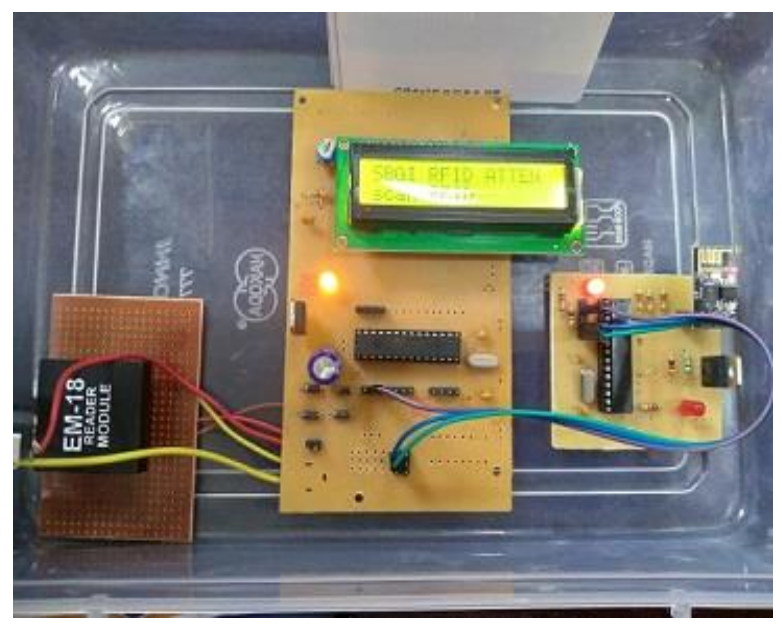

Step 2: 
International Journal of Engineering Applied Sciences and Technology, 2021

Vol. 6, Issue 1, ISSN No. 2455-2143, Pages 353-357

Published Online May 2021 in IJEAST (http://www.ijeast.com)

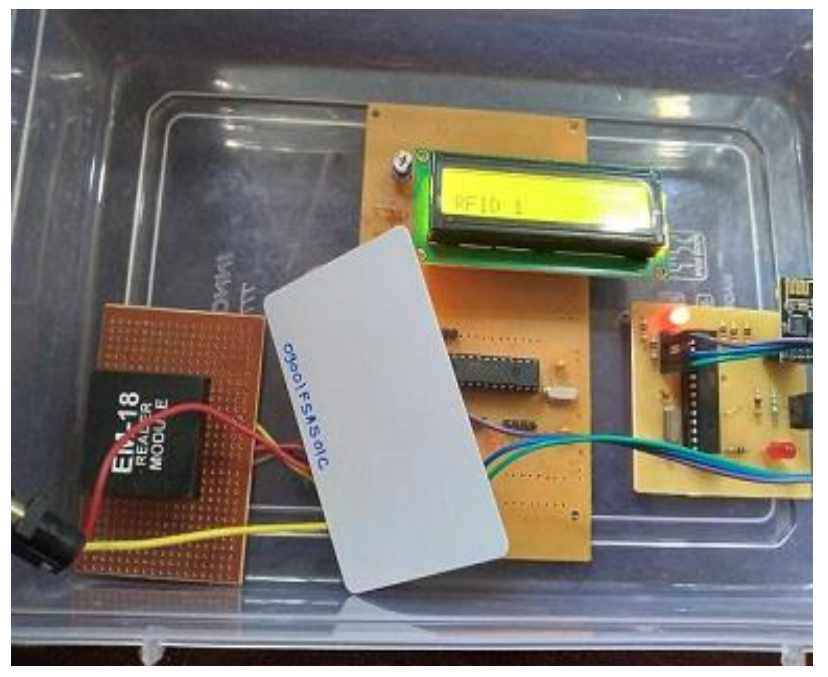

Step 3:

IOT Interface

IOT BASED ATTENDANCE SYSTEM

CLEAR

\begin{tabular}{|c|c|c|c|}
\hline $\mathrm{min}$ & nvit & mousoson & nove \\
\hline $25-25-200$ & Date & $a$ & suoxm! \\
\hline
\end{tabular}

Step 4:

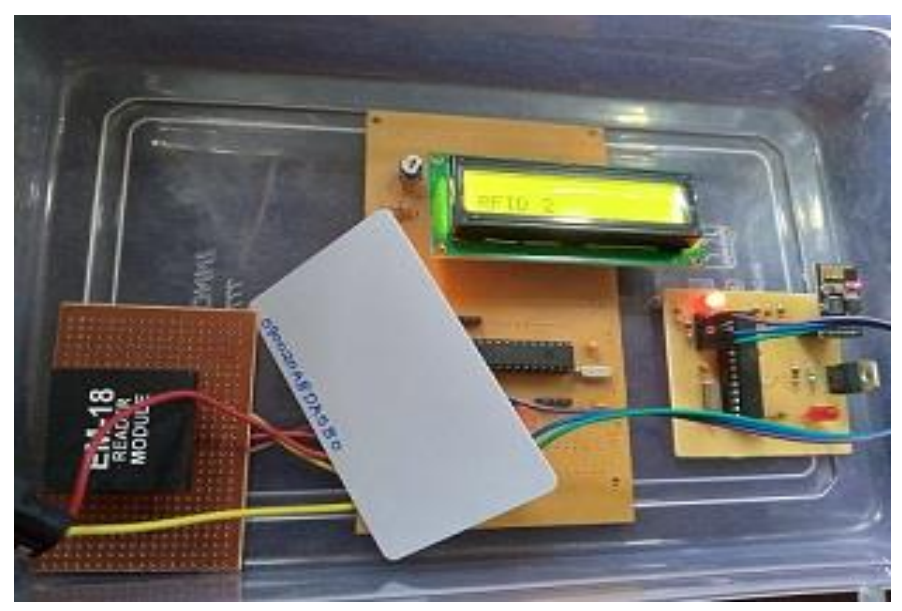

Step 5:

IOT Interface

\section{IOT BASED ATTENDANCE SYSTEM}

CLEAR

\begin{tabular}{|c|c|c|c|}
\hline amit & TMA & nouno & sиме \\
\hline $25-06-2021$ & 113056 & on & stuen: \\
\hline $25-00-2021$ & $n \geq 0$ & $\infty$ & sucenrz \\
\hline
\end{tabular}

Step 6:

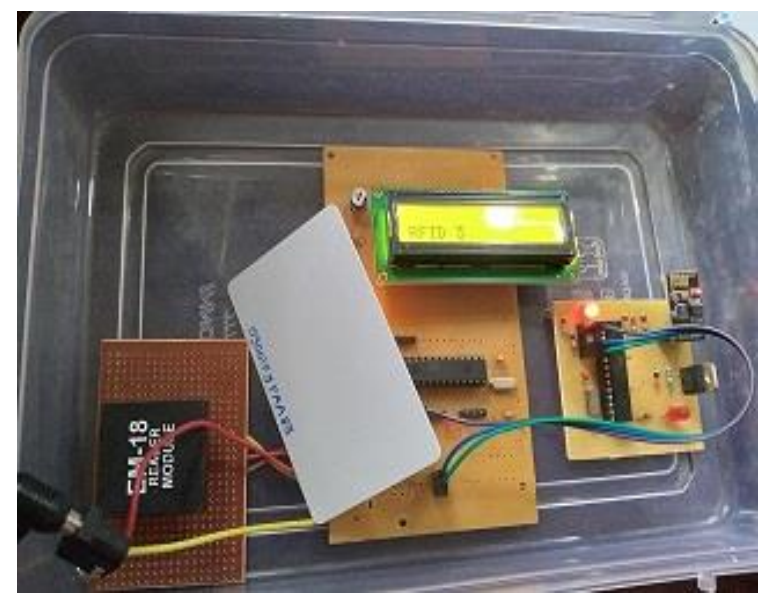

Step 7:

IOT Interface

\section{IOT BASED ATTENDANCE SYSTEM}

CLEAR

\begin{tabular}{|c|c|c|c|}
\hline oart & Twe & rou No & NaME \\
\hline $26-96-2 \mathrm{xt}$ & ค셔 & 9 & shever \\
\hline $25-\infty-2,-30$ & $n=282$ & 02 & shever \\
\hline $25-\infty-2000$ & ressiss & os & stuevess \\
\hline
\end{tabular}

Step 8: 


\section{IOT BASED ATTENDANCE SYSTEM}

CLEAR

onT

nue

nouns

naw

\section{CONCLUSION}

The Same System can be applied to corporate offices, government offices, schools, colleges, in online exam centers. Multifunction smart ID cards with RFID offer several easy and cost-effective ways for campuses to raise the level of protection and quality of education. Today's smart access card technologies provide superior range and read performance so that educational institutions can improve efficiency and security at multiple levels.

\section{REFERENCES}

1. RFID and IOT for Attendance Monitoring System Joseph Dedy Irawan 1,*, Emmalia Adriantantri 2, and Akh Farid1 1 Informatics, National Institute of Technology, Raya Karanglo Km.2 Malang 65153, Indonesia 2 Industrial Engineering, National Institute of Technology, Raya Karanglo Km.2 Malang 65153, Indonesia

2. RFID Based Attendance System using IoT B. Nandhakumar, K. Naveen Kumar, M. Vijay, A. Sriram Department of Electronics and Communication Engineering, Arasu Engineering College, Kumbakonam - 612501. India.

3. Sri Madhu BM, Kavya K, Devansh. IoT based automatic attendance management system, IEEE International Conference on Current Trends in Computer, Electrical, Electronics and Communication, September 2017. DOI: 10.1109/CTCEEC.2017.8455099

4. Hassan S, Ertan Z, Ghassan AH. New anti-collision protocol for RFID - Based Student Attendance system. 2nd International Symposium on Multidisciplinary Studies and Innovative Technologies (ISMSIT). October 2018. DOI: 10.1109/ISMSIT.2018.8567278

5. Rupak Kumar, Harshi Solanki, "RFID an Effective Authentication and Attendance System", International Journal of Advanced Research in Computer Science and Software Engineering, Volume 3, Issue 6, June 2013, page no.1234-1236, ISSN: 2277 128X 